\title{
Analytic Solutions for Full Operating Range Single-Side ZVS Modulation of Dual Active Bridge Converters
}

\author{
Michael Saegmueller \\ Research High Voltage Electric Powertrain \\ $B M W A G$ \\ Garching, Germany \\ michael.saegmueller@tum.de
}

\author{
Rolf Witzmann \\ Chair of High Voltage Engineering and Switchgear Technology \\ Technical University Munich \\ Munich, Germany \\ rolf.witzmann@tum.de
}

\author{
Christoph Hackl \\ Department of Electrical Engineering and Information Technology \\ Munich University of Applied Sciences \\ Munich, Germany \\ christoph.hackl@hm.edu
}

\author{
René Richter \\ Research High Voltage Electric Powertrain \\ $B M W A G$ \\ Garching, Germany \\ rene.richter@bmw.de
}

TABLE I

Abstract-This paper presents analytic solutions for an optimal modulation scheme featuring low switching losses for a bidirectional single-phase dual active bridge (DAB) DC-DC converter used for charging high-voltage batteries of electric vehicles. The proposed modulation scheme facilitates zero-voltage switching (ZVS) for either the primary- or secondary-side full bridge of the DAB converter throughout the whole operating range while maintaining low conduction losses. The expressions are derived based on the current required for an ideal ZVS transition and enable for a direct computation of the respective optimal modulation parameters.

Index Terms-Battery charger, dual active bridge, zero-voltage switching

\section{INTRODUCTION}

The increasing spread of renewable energy sources, such as photovoltaic systems and fuel cells, as well as electrified vehicles rises the need for advanced power electronic systems. In particular, the importance of their efficiency and power density increases. A dual active bridge (DAB) converter is used as an isolated DC-DC stage in an electric vehicle battery charger. Despite the galvanic isolation it enables very high efficiencies [1] and additionally exhibits a bidirectional power flow capability [2]. The specification of the considered DAB converter is summarized in Tab. I.

Various modulation schemes for DAB converters have been presented in literature, featuring low conduction and/or switching losses. For power MOSFETs, the latter is commonly achieved by applying the principle of zero-voltage switching (ZVS) [3]. Additionally, the proposed modulation schemes enable for an analytic calculation of the respective optimal modulation parameters.

In [4], a modulation scheme is presented which aims at operating the DAB with lowest conduction losses and facilitates an analytical calculation of the respective optimal modulation parameters. [5] extends this approach by considering the switching frequency as an additional modulation parameter. However, ZVS cannot be achieved throughout substantial regions of the operating range with these modulation schemes.

In [7], a modulation scheme is proposed which ensures full operating range ZVS for all switching devices. Though, this approach relies on hardware modifications as it requires two additional commutation inductors to be inserted into the DAB circuit. These inductors beneficially influence the switching losses but in turn increase the RMS currents and hence the conduction losses.

A modulation scheme which inherently features ZVS for every switching device is presented in [6]. However, ZVS cannot be maintained throughout the whole operating range as it is lost at some transitions between different switching modes.

Finally, in [1], a modulation scheme is used, which enables for primary-side ZVS for all respective operating points. Simultaneously, secondary-side ZVS is ensured by one additional commutation inductor. However, this approach is based on a transformation applied to the DAB model which renders a direct calculation of the optimal modulation parameters infeasible. 


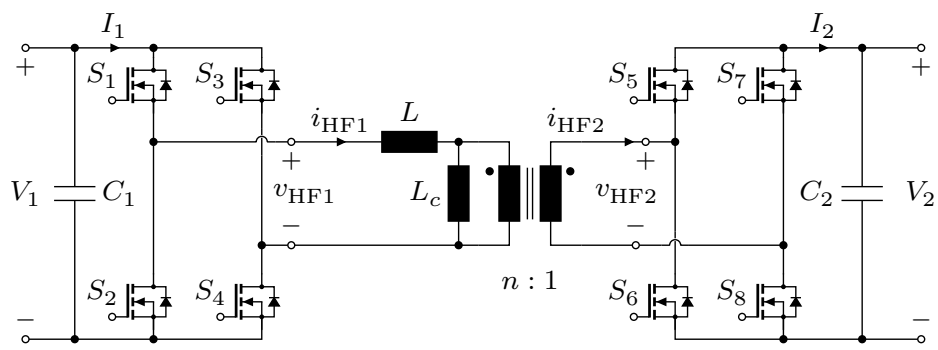

(a)

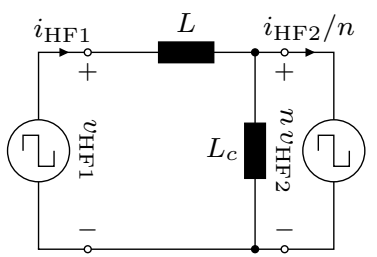

(b)

Fig. 1: (a) DAB converter topology; (b) simplified lossless DAB model.

This paper derives analytic expressions which facilitate a direct computation of an optimal set of modulation parameters. The parameters are determined such that full operating range $\mathrm{ZVS}$ is achieved for the primary-side switches. Analogously to the approach presented in [1], a commutation inductor $L_{\mathrm{c}}$ is added to ensure ZVS for the secondary-side switches. For the sake of clarity, the determination of the optimal modulation parameters is explained solely for positive power transfer and primary-side ZVS, however, the presented equations are also valid for negative power transfer and/or secondary-side ZVS.

\section{DAB MODEL AND MODULATION SCHEMES}

The topology of the dual active bridge converter is shown in Fig. 1(a). Assuming that the components are lossless, the most simple DAB model shown in Fig. 1(b) can be obtained. The two full bridges can be thereby considered as voltage sources which apply the square-wave voltages $v_{\mathrm{HF} 1}(t)$ and $n v_{\mathrm{HF} 2}(t)$ to the high-frequency (HF) AC-link circuit. The latter contains a HF transformer with a turns ratio of $n: 1$, the series inductor $L$ and the commutation inductor $L_{\mathrm{c}}$, which only introduces reactive power and does not contribute to the power transfer. As mentioned before, the latter is added to achieve ZVS for the secondary-side switches. In discrete time intervals, the current $i_{L}(t)$ through $L$ can be described by

$$
i_{L}\left(t_{\mathrm{i}}\right)=i_{L}\left(t_{\mathrm{i}-1}\right)+\frac{v_{\mathrm{HF} 1}\left(t_{\mathrm{i}}\right)-n v_{\mathrm{HF} 2}\left(t_{\mathrm{i}}\right)}{L}\left(t_{\mathrm{i}}-t_{\mathrm{i}-1}\right) .
$$

With to full bridges, twelve different switching modes can be realized, each characterized by its individual sequence of rising and falling edges of the voltages $v_{\mathrm{HF} 1}$ and $v_{\mathrm{HF} 2}$. For an efficient DAB operation with low conduction losses, only four different switching modes should be thereof applied [4]. A description of these four modes as well as exemplary waveforms can be found in [5].

To determine the power flow of the DAB converter, the combination of the three modulation parameters $\varphi$ (phase shift between the rising edges of $v_{\mathrm{HF} 1}$ and $v_{\mathrm{HF} 2}$ ), $\tau_{1}$ and $\tau_{2}$ (pulse widths of the voltages $v_{\mathrm{HF} 1}$ and $v_{\mathrm{HF} 2}$, respectively) is used (cf. Fig. 2(a)). These parameters can be adjusted in the range $-0.5 \leq \varphi \leq 0.5$ and $0 \leq \tau \leq 0.5$.

Three different modulation schemes can be employed with the four switching modes mentioned before, which are:

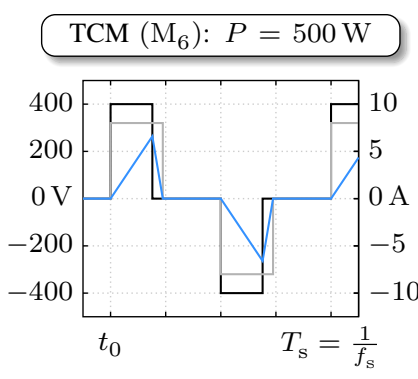

(a)

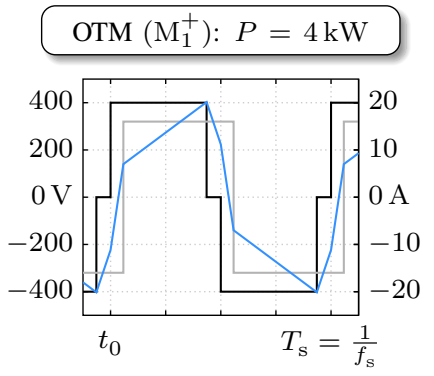

(c)

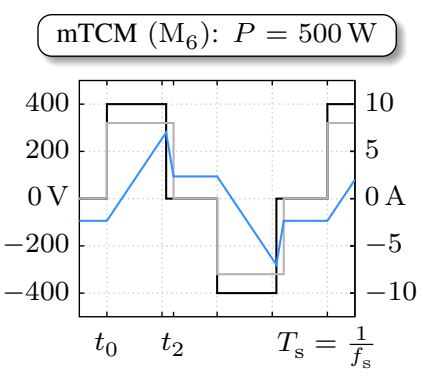

(b)

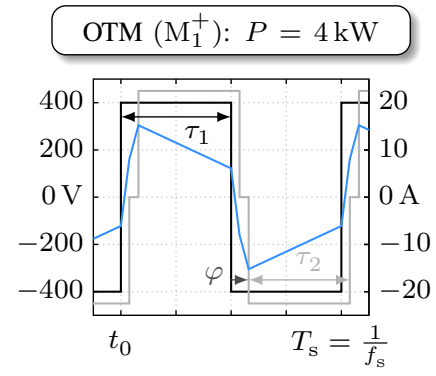

(d)
Fig. 2: Exemplary waveforms for (a) the triangular current mode (TCM), (b) modified triangular current mode (mTCM), (c) optimal transition mode for buck mode operation and (d) optimal transition mode for boost-mode operation; $v_{\mathrm{HF} 1}(-), n v_{\mathrm{HF} 2}(-)$ and $i_{L}(-)$.

1) Modified Triangular Current Mode (mTCM): Used for low power levels $P \leq P_{\max }^{\mathrm{mtcm}}$. For this modulation scheme, switching modes $\mathrm{M}_{5}$ and $\mathrm{M}_{6}$ are applied.

2) (Modified) Optimal Transition Mode (OTM): OTM is employed for medium powers, i.e. $P_{\max }^{\mathrm{mtcm}}<P \leq P_{\max }^{\mathrm{otm}}$. This modulation scheme can be realized by switching mode $\mathrm{M}_{1}^{+}$for power levels $P>0$ and $\mathrm{M}_{1}^{-}$for $P<0$.

3) Phase Shift Mode (PSM): Used for high power levels $P_{\max }^{\text {otm }}<P \leq P_{\max }$. For this modulation scheme, switching modes $\mathrm{M}_{1}^{+}$and $\mathrm{M}_{1}^{-}$are used as well. However, $\tau_{1}=\tau_{2}=0.5$ always applies for PSM, i.e. the power is solely controlled by the phase-shift $\varphi$.

\section{ANALYTICAL DETERMINATION OF OPTIMAL MODULATION PARAMETERS FOR PRIMARY-SIDE ZVS}

To facilitate an anlytic calculation of the optimal control variables $\tau_{1, \mathrm{opt}}, \tau_{2, \mathrm{opt}}$ and $\varphi_{\mathrm{opt}}$, analytical expressions for the 
currents $i_{L}\left(t_{\mathrm{i}}\right)$ at the respective switching instants and for the transferred power

$$
P=\frac{2}{T_{\mathrm{s}}} \int_{t_{0}}^{t_{0}+T_{\mathrm{s}} / 2} i_{L}(t) v_{\mathrm{HF} 1}(t) \mathrm{d} t
$$

are needed. The respective switching instants $t_{\mathrm{i}}$ needed for the calculation of the currents and for the evaluation of (2) can be expressed by the modulation parameters introduced before. Expressions for every switching instant as well as solutions for the phase-shift $\varphi$ for all switching modes were proposed in [5] and have been obtained by reformulating (2) with respect to $\varphi$.

\section{A. ZVS Requirements}

In order to avoid losses caused by hard-switching transitions, the principle of ZVS is usually applied for MOSFETs. Ideal soft-switching requires an impressed current of an inductive component which charges/discharges the MOSFETs output capacitance $C_{\text {oss }}$ within a bridge leg, such as $S_{1}$ and $S_{2}$ in Fig. 1(a). Two conditions are required for a complete zerovoltage transition [3]:

1) If $S_{1}$ is turned off and $S_{2}$ is turned on, which leads to the charging of $C_{\mathrm{oss}, 1}$ and the discharging of $C_{\mathrm{oss}, 2}$, the current must flow out of the bridge leg, i.e. $i_{\mathrm{HF} 1}>0$. For a transition from $S_{2}$ to $S_{1} i_{\mathrm{HF} 1}<0$, is required.

2) Additionally, the current at the respective switching instant must be large enough, i.e. it must provide enough charge $Q_{\text {oss }}$ to fully charge/discharge the MOSFET output capacitance. This can be expressed by the energy balance

$$
\frac{1}{2} L I_{\mathrm{zvs}}^{2} \geq Q_{\mathrm{oss}}\left(V_{\mathrm{DC}}\right) \cdot V_{\mathrm{DC}}
$$

If an additional parasitic layout capacitance $C_{\mathrm{par}}$ is considered, $\frac{1}{2} C_{\mathrm{par}} V_{\mathrm{DC}}^{2}$ has to be added to the right-hand side of (3). Finally, the current required for a complete ZVS transition can be obtained by reformulating (3) with respect to $I_{\mathrm{zvs}}$.

The optimal modulation parameters are determined in such a way, that the energy balance of (3) is fulfilled for every switching instant of $v_{\mathrm{HF} 1}$, i.e. primary-side ZVS is achieved throughout the whole operating range. For the calculation of the optimal modulation parameters, the following currents

$$
\begin{aligned}
& I_{1}=-I_{\mathrm{zvs}} \\
& I_{2}=I_{\mathrm{zvs}}
\end{aligned}
$$

are defined. Note that for secondary-side ZVS the currents must be interchanged.

\section{B. Closed-form solutions for optimal $\tau_{1}, \tau_{2}$ and $\varphi$}

In order to reduce the number of equations needed to fully describe the derivation of the optimal modulation parameters, a transformation according to

$$
\begin{aligned}
\left(V_{\mathrm{A}},\right. & \left.V_{\mathrm{B}}, \tau_{\mathrm{A}}, \tau_{\mathrm{B}}, I_{\mathrm{A}}, I_{\mathrm{B}}\right)^{\top} \\
& =\left\{\begin{array}{lll}
\left(V_{1}, n V_{2}, \tau_{1}, \tau_{2}, I_{1}, I_{2}\right)^{\top} & \forall & V_{1} \leq n V_{2} \\
\left(n V_{2}, V_{1}, \tau_{2}, \tau_{1}, I_{2}, I_{1}\right)^{\top} & \forall & V_{1}>n V_{2}
\end{array}\right.
\end{aligned}
$$

is applied. Note that by these transformation, voltages, currents and pulse widths are interchanged.

1) Modified Triangular Current Mode: As mentioned before, $\mathrm{mTCM}$ is used for low power levels. In contrast to normal TCM, $\tau_{\mathrm{A}}$ and $\tau_{\mathrm{B}}$ are determined such, that the current $i_{L}$ is offset during the freewheeling intervals, i.e. $\left|i_{L}(t)\right|=I_{\mathrm{zvs}}>0$ for $v_{\mathrm{HF} 1}(t)=v_{\mathrm{HF} 2}(t)=0$ (cf. Fig. 2(a) and Fig. 2(b)). By applying (5) to the currents at the switching instants $t_{0}$ and $t_{2}$ of mode $\mathrm{M}_{6}$ the following equations can be obtained

$$
\begin{aligned}
& i_{L}\left(t_{0}\right)=I_{\mathrm{B}}=\frac{V_{\mathrm{B}} \tau_{\mathrm{B}}^{2}\left(V_{\mathrm{A}}-V_{\mathrm{B}}\right)+f_{\mathrm{S}} L P}{2 f_{\mathrm{s}} L V_{\mathrm{B}} \tau_{\mathrm{B}}}, \\
& i_{L}\left(t_{2}\right)=I_{\mathrm{A}}=\frac{V_{\mathrm{B}} \tau_{\mathrm{B}}-V_{\mathrm{A}} \tau_{\mathrm{A}}}{2 f_{\mathrm{S}} L} .
\end{aligned}
$$

For mode $\mathrm{M}_{5}$ the expressions on the right-hand sides of (6) and (7) must be interchanged. To calculate the respective optimal pulse widths, these equations must be solved for $\tau_{\mathrm{A}}$ and $\tau_{\mathrm{B}}$. However, the expression for $\tau_{\mathrm{B}}$ is only valid for $V_{\mathrm{A}} \neq V_{\mathrm{B}}$, i.e. $V_{1} \neq n V_{2}$. An alternative solution can be obtained by applying $V_{\mathrm{A}}=V_{\mathrm{B}}$ to (6) and reformulating with respect to $\tau_{\mathrm{B}}$. Finally, the expressions needed to calculate the respective optimal modulation parameters are

$$
\begin{aligned}
& \forall \quad|P| \leq P_{\max }^{\operatorname{mtcm}} \\
& \left\{\begin{array}{l}
\tau_{\mathrm{B}}=\left\{\begin{array}{l}
\sqrt{\left(\frac{f_{\mathrm{s}} L I_{\mathrm{B}}}{V_{\mathrm{A}}-V_{\mathrm{B}}}\right)^{2}-\frac{f_{\mathrm{s}} L|P|}{V_{\mathrm{B}}\left(V_{\mathrm{A}}-V_{\mathrm{B}}\right)}} \\
+\frac{f_{\mathrm{s}} L I_{\mathrm{B}}}{V_{\mathrm{A}}-V_{\mathrm{B}}} \quad \forall V_{1} \neq n V_{2} \\
\frac{|P|}{2 V_{\mathrm{B}}\left|I_{\mathrm{B}}\right|} \quad \forall \quad V_{1}=n V_{2}
\end{array}\right. \\
\tau_{\mathrm{A}}=\frac{V_{\mathrm{B}} \tau_{\mathrm{B}}-2 f_{\mathrm{s}} L I_{\mathrm{A}}}{V_{\mathrm{A}}} \\
\varphi=\operatorname{sgn}\left(V_{1}-n V_{2}\right) \frac{\tau_{\mathrm{B}}-\tau_{\mathrm{A}}}{2}+\frac{f_{\mathrm{s}} L P}{2 V_{\mathrm{A}} V_{\mathrm{B}} \tau_{\mathrm{B}}}
\end{array} .\right.
\end{aligned}
$$

As mentioned in [5], the duration of the freewheeling interval decreases with increasing power $P$ until it becomes zero at the highest possible output power $P_{\max }^{\operatorname{mtcm}}$. An expression for $P_{\max }^{\text {mtcm }}$ can thus be found by inserting $\tau_{\mathrm{B}}$ into $\tau_{\mathrm{A}}$ and solving $\tau_{\mathrm{A}}=0.5$ for $P$. This yields

$$
\begin{aligned}
P_{\max }^{\operatorname{mtcm}}=\left(V_{\mathrm{A}}\right. & \left.+4 f_{\mathrm{s}} L I_{\mathrm{A}}\right) \\
& \cdot\left(I_{\mathrm{B}}+\frac{\left(V_{\mathrm{A}}+4 f_{\mathrm{s}} L I_{\mathrm{A}}\right)\left(V_{\mathrm{B}}-V_{\mathrm{A}}\right)}{4 f_{\mathrm{s}} L V_{\mathrm{B}}}\right) .
\end{aligned}
$$

In contrast to standard TCM, switching modes $\mathrm{M}_{5}$ and $\mathrm{M}_{6}$ are not only applied for $V_{1}<n V_{2}$ and $V_{1}>n V_{2}$, respectively. Corresponding operating points are indicated by the shaded area in Fig. 5(a). If (8) and (9) are evaluated for the operating points mentioned before, incorrect values for $\tau_{\mathrm{A}}, \tau_{\mathrm{B}}$ and $\varphi$ result and $P_{\max }^{\mathrm{mtcm}}$ becomes negative, which is unfeasible. 
However, appropriate values for $\tau_{\mathrm{A}}, \tau_{\mathrm{B}}, \varphi$ and $P_{\max }^{\mathrm{mtcm}}$ can be obtained. This results in the modified equations

$$
\begin{aligned}
& \forall \quad|P| \leq P_{\max }^{\operatorname{mtcm}} \\
& \left\{\begin{array}{l}
\tau_{\mathrm{A}}=\left\{\begin{array}{l}
-\sqrt{\left(\frac{f_{\mathrm{s}} L I_{\mathrm{A}}}{V_{\mathrm{B}}-V_{\mathrm{A}}}\right)^{2}-\frac{f_{\mathrm{s}} L|P|}{V_{\mathrm{A}}\left(V_{\mathrm{B}}-V_{\mathrm{A}}\right)}} \\
+\frac{f_{\mathrm{s}} L I_{\mathrm{A}}}{V_{\mathrm{B}}-V_{\mathrm{A}}} \quad \forall V_{1} \neq n V_{2}
\end{array}\right. \\
\tau_{\mathrm{A}}=\frac{V_{\mathrm{A}} \tau_{\mathrm{A}}-2 f_{\mathrm{s}} L I_{\mathrm{B}}}{V_{\mathrm{B}}} \\
\varphi=\operatorname{sgn}\left(V_{1}-n V_{2}\right) \frac{\tau_{\mathrm{B}}-\tau_{\mathrm{A}}}{2}+\frac{f_{\mathrm{s}} L P}{2 V_{\mathrm{A}} V_{\mathrm{B}} \tau_{\mathrm{A}}} .
\end{array}\right.
\end{aligned}
$$

for the calculation respective modulation parameters. For according operating points the highest possible power for mTCM can be obtained from

$$
\begin{aligned}
& P_{\max }^{\operatorname{mtcm}}=\left(V_{\mathrm{A}}+4 f_{\mathrm{s}} L I_{\mathrm{B}}\right) \\
& \cdot\left(I_{\mathrm{A}}+\frac{\left(V_{\mathrm{B}}+4 f_{\mathrm{s}} L I_{\mathrm{B}}\right)\left(V_{\mathrm{A}}-V_{\mathrm{B}}\right)}{4 f_{\mathrm{s}} L V_{\mathrm{A}}}\right) .
\end{aligned}
$$

It should be noted that for the special case $I_{1}=I_{2}=0$, i.e. standard TCM is applied, the equations of (8), (10), (9) and (11) transform into the ones presented in [5].

2) Optimal Transition Mode: OTM is employed for medium power levels and is realized with switching mode $\mathrm{M}_{1}^{+}$. Exemplary waveforms for OTM in buck and boost operation are shown in Fig. 2(c) and Fig. 2(d), respectively. As mentioned before, $\tau_{\mathrm{A}}=0.5$ always applies for $P \geq P_{\max }^{\operatorname{mtcm}}$. A closed-form expression for the calculation of the optimal pulse width $\tau_{\mathrm{B}}$ regarding minimum conduction losses has been derived in [5] and is given by

$$
\begin{aligned}
& e_{0}=V_{\mathrm{A}}^{2}+V_{\mathrm{B}}^{2} \\
& e_{1}=\left(4 f_{\mathrm{s}} L P e_{0}\right)^{2}\left(8 V_{\mathrm{A}}^{2}-V_{\mathrm{B}}^{2}\right)+6 V_{\mathrm{A}}^{6} V_{\mathrm{B}}^{2}\left(e_{0}+3 V_{\mathrm{A}}^{2}\right) \\
& -3 f_{\mathrm{s}} L|P| V_{\mathrm{A}}^{3} V_{\mathrm{B}}\left(32 V_{\mathrm{A}}^{4}+28 V_{\mathrm{A}}^{2} V_{\mathrm{B}}^{2}+5 V_{\mathrm{B}}^{4}\right) \\
& e_{2}=6 \sqrt{3} V_{\mathrm{A}} V_{\mathrm{B}}^{2}\left[f _ { \mathrm { s } } L | P | \left[V_{\mathrm{A}}^{7} V_{\mathrm{B}}^{5}-64\left(f_{\mathrm{s}} L|P| e_{0}\right)^{3}\right.\right. \\
& +V_{\mathrm{A}} V_{\mathrm{B}} f_{\mathrm{s}} L|P|\left(4 e_{0}-3 V_{\mathrm{B}}^{2}\right) \\
& \left.\left.\cdot\left(8 f_{\mathrm{s}} L|P| e_{0}^{2}-V_{\mathrm{A}}^{3} V_{\mathrm{B}}\left(e_{0}+\frac{9}{4} V_{\mathrm{B}}^{2}\right)\right)\right]\right]^{\frac{1}{2}} \\
& e_{3}=f_{\mathrm{s}} L|P|\left(f_{\mathrm{s}} L|P| e_{0}-\frac{V_{\mathrm{A}}^{3} V_{\mathrm{B}}}{2}\right) \\
& \cdot\left(e_{0}-\frac{3}{4} V_{\mathrm{B}}^{2}\right)+\left(\frac{V_{\mathrm{A}}^{3} V_{\mathrm{B}}}{4}\right)^{2} \\
& e_{4}=4\left[\frac{4 f_{\mathrm{s}} L|P| e_{0}^{2}}{V_{\mathrm{A}} V_{\mathrm{B}}}-e_{0}^{2}+V_{\mathrm{B}}^{2}\left(e_{0}-\frac{3}{4} V_{\mathrm{B}}^{2}\right)\right]
\end{aligned}
$$

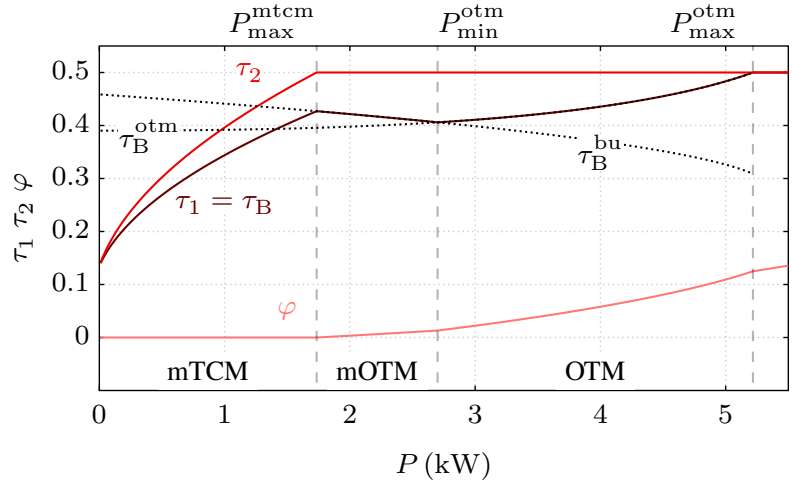

(a)

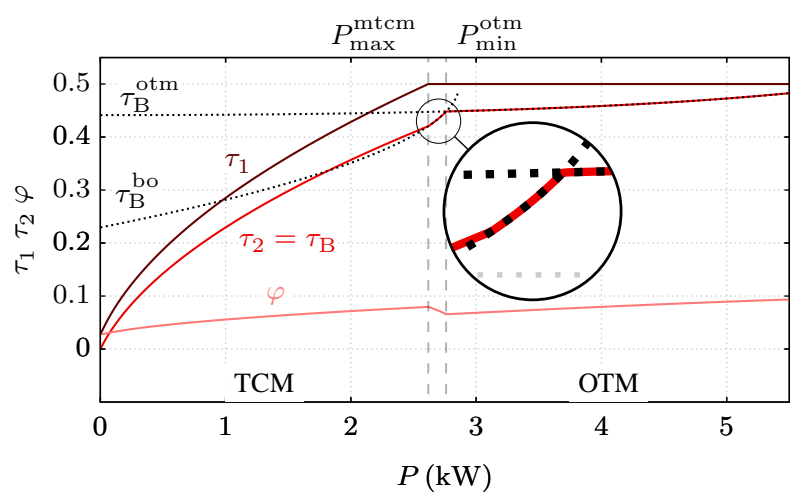

(b)

Fig. 3: Trajectories of the optimal control parameters $\tau_{1}, \tau_{2}$ and $\varphi$ for (a) buck-mode operation, $V_{1}=V_{\mathrm{B}}=400 \mathrm{~V}, n V_{2}=V_{\mathrm{A}}=320 \mathrm{~V}$, and (b) boost-mode operation, $V_{1}=V_{\mathrm{A}}=400 \mathrm{~V}, n V_{2}=V_{\mathrm{B}}=450 \mathrm{~V}$. The trajectories of $\tau_{\mathrm{B}}^{\mathrm{otm}}, \tau_{\mathrm{B}}^{\mathrm{bu}}$ and $\tau_{\mathrm{B}}^{\mathrm{bo}}$, which are required for the determination of $\tau_{\mathrm{B}}$ for mOTM intervals are additionally shown.

$$
\begin{aligned}
e_{5}= & \sqrt[3]{f_{\mathrm{s}} L|P|\left(e_{2}-e_{1}\right)+2 V_{\mathrm{A}}^{9} V_{\mathrm{B}}^{3}} \\
e_{6}= & \sqrt[3]{2} e_{0} \frac{32 e_{3}+e_{5}^{2} \sqrt[3]{2}}{V_{\mathrm{A}} V_{\mathrm{B}} e_{5}} \\
\tau_{\mathrm{B}}^{\text {otm }}= & \frac{1}{4}\left(\frac{\sqrt{2 e_{6}-e_{4}}}{\sqrt{3} e_{0}}+\frac{V_{\mathrm{A}}^{2}}{e_{0}}+1\right)-\left[-\frac{e_{6}+e_{4}}{24 e_{0}^{2}}\right. \\
& \left.+\frac{\sqrt{3} V_{\mathrm{B}}\left(V_{\mathrm{A}} V_{\mathrm{B}}^{3}\left(e_{0}+V_{\mathrm{A}}^{2}\right)-8 f_{\mathrm{s}} L|P| e_{0}^{2}\right)}{8 e_{0}^{2} V_{\mathrm{A} \sqrt{2 e_{6}-e_{4}}}}\right]^{\frac{1}{2}}
\end{aligned}
$$

An expression for the calculation of the maximum transferable power

$$
P_{\max }^{\text {otm }}=\frac{V_{\mathrm{B}}\left(\left(V_{\mathrm{A}}^{2}-V_{\mathrm{B}}^{2}+V_{\mathrm{B}} \sqrt{V_{\mathrm{B}}^{2}-V_{\mathrm{A}}^{2}}\right)\right)}{4 f_{\mathrm{s}} L V_{\mathrm{A}}}
$$

has been presented in [5] as well. However, primary-side ZVS cannot be achieved inherently in OTM for all respective operating points. Especially for power levels slightly above 
$P_{\max }^{\mathrm{mtcm}} \mathrm{ZVS}$ is lost if standard OTM is used. Thus, a different modulation scheme must be applied for these operating points.

3) Modified Optimal Transition Mode: For the operating points mentioned before, a modified version of the optimal transition mode named mOTM is proposed. To guarantee primary-side ZVS for OTM, $i_{L}\left(t_{0}\right) \leq-I_{\text {zvs }}$ must be fulfilled. Distinguishing whether the DAB operates in buck or in boost mode, $i_{L}\left(t_{0}\right)=-I_{\mathrm{zvs}}$ must be either solved for $\tau_{1}$ or $\tau_{2}$, respectively. By applying (5) to the solutions, one obtains

$$
\begin{aligned}
\tau_{\mathrm{B}}^{\mathrm{bu}}= & \frac{1}{2\left(\left(V_{\mathrm{A}}+V_{\mathrm{B}}\right)^{2}+V_{\mathrm{A}}^{2}\right)}\left[\left[2 V_{\mathrm{A}}^{3}\left(V_{\mathrm{A}}+V_{\mathrm{B}}\right)-8 f_{\mathrm{s}} L V_{\mathrm{A}}^{2}\right.\right. \\
& \left.\cdot\left(|P| \frac{\left(V_{\mathrm{A}}+V_{\mathrm{B}}\right)^{2}+V_{\mathrm{A}}^{2}}{V_{\mathrm{A}} V_{\mathrm{B}}}+I_{\mathrm{B}}\left(2 f_{\mathrm{s}} L I_{\mathrm{B}}+V_{\mathrm{B}}\right)\right)\right]^{\frac{1}{2}} \\
& \left.+V_{\mathrm{A}}\left(2 V_{\mathrm{A}}+V_{\mathrm{B}}\right)-4 f_{\mathrm{s}} L I_{\mathrm{B}}\left(V_{\mathrm{A}}+V_{\mathrm{B}}\right)\right] \\
\tau_{\mathrm{B}}^{\mathrm{bo}}= & \frac{1}{2}-\sqrt{\frac{1}{4}-\frac{2 f_{\mathrm{s}} L|P|}{V_{\mathrm{A}} V_{\mathrm{B}}}-\left(\frac{4 f_{\mathrm{s}} L I_{\mathrm{A}}+V_{\mathrm{A}}}{2 V_{\mathrm{B}}}\right)^{2}} \cdot
\end{aligned}
$$

It can be seen, that either $I_{\mathrm{B}}$ or $I_{\mathrm{A}}$ is exclusively considered for the calculation of $\tau_{\mathrm{B}}$, which always corresponds to $I_{1}$. For secondary-side ZVS, the expressions for $\tau_{\mathrm{B}}^{\text {bu }}$ and $\tau_{\mathrm{B}}^{\text {bo }}$ must be interchanged, i.e. $I_{2}$ is always considered.

Depending on whether the DAB operates in buck or in boost mode, a value for $P_{\mathrm{otm} \text {,min }}$ could be obtained by solving $\tau_{\mathrm{B}}^{\text {otm }}=\tau_{\mathrm{B}}^{\text {bu }}$ or $\tau_{\mathrm{B}}^{\text {otm }}=\tau_{\mathrm{B}}^{\text {bo }}$ with respect to $P$. However, no closed-form expression has been found due to the complexity of the result. Thus, a straightforward approach is presented to determine the respective pulse width $\tau_{\mathrm{B}}$ which facilitates primary-side ZVS throughout the whole OTM. This approach is shown in Fig. 3. It is based on a comparison of the trajectories of $\tau_{\mathrm{B}}^{\text {otm }}$ and $\tau_{\mathrm{B}}^{\text {bu }}$ and $\tau_{\mathrm{B}}^{\text {bo }}$, respectively. The closedform expressions needed to calculate the respective modulation parameters for OTM are finally given by

$$
\left\{\begin{aligned}
\forall \quad & P_{\max }^{\mathrm{mtcm}}<|P| \leq P_{\max }^{\mathrm{otm}} \\
\tau_{\mathrm{A}}= & \frac{1}{2} \\
\tau_{\mathrm{B}}= & \left\{\begin{array}{l}
\min \left(\max \left(\tau_{\mathrm{B}}^{\mathrm{otm}}, \tau_{\mathrm{B}}^{\mathrm{bu}}\right), \frac{1}{2}\right) \quad \forall \quad V_{1}>n V_{2} \\
\min \left(\min \left(\tau_{\mathrm{B}}^{\mathrm{otm}}, \tau_{\mathrm{B}}^{\mathrm{bo}}\right), \frac{1}{2}\right) \quad \forall \quad V_{1} \leq n V_{2}
\end{array}\right. \\
\varphi= & \operatorname{sgn}\left(n V_{2}-V_{1}\right) \frac{\tau_{\mathrm{A}}-\tau_{\mathrm{B}}}{2}+\operatorname{sgn}(P)\left(\frac{1}{4}-\frac{1}{2}\right. \\
& \left.\cdot \sqrt{\frac{1}{4}-\frac{2 f_{\mathrm{s}} L|P|}{V_{\mathrm{A}} V_{\mathrm{B}}}-\left(\tau_{\mathrm{A}}-\frac{1}{2}\right)^{2}-\left(\tau_{\mathrm{B}}-\frac{1}{2}\right)^{2}}\right) .
\end{aligned}\right.
$$

4) Phase Shift Mode: PSM is used at high power levels and inherently features ZVS. The respective modulation parameters can be calculated as follows

$$
\begin{aligned}
\forall \quad P_{\max }^{\mathrm{otm}}<|P| \leq P_{\max } \\
\left\{\begin{array}{l}
\tau_{\mathrm{A}}=\tau_{\mathrm{B}}=\frac{1}{2} \\
\varphi=\operatorname{sgn}(P) \frac{1}{4}\left(1-\sqrt{1-\frac{8 f_{\mathrm{s}} L|P|}{V_{\mathrm{A}} V_{\mathrm{B}}}}\right) .
\end{array}\right.
\end{aligned}
$$

Finally, the procedure to analytically determine the respective optimal modulation parameters for given $n, L, V_{1}, V_{2}$ and $P$ is illustrated in Fig. 4 as flow chart.

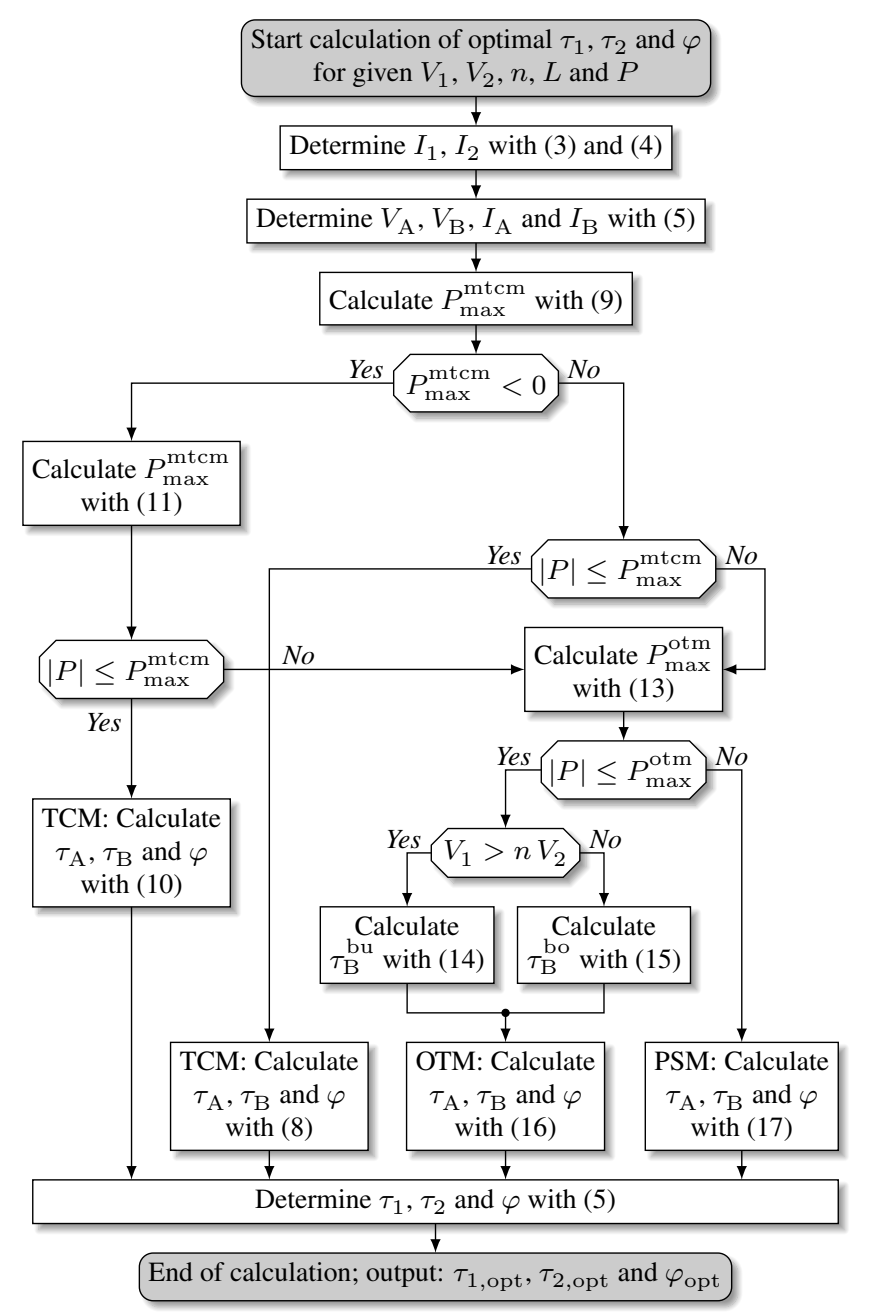

Fig. 4: Procedure to analytically determine optimal control parameters $\tau_{1, \text { opt }}$, $\tau_{2, \text { opt }}$ and $\varphi_{\text {opt }}$ for given converter parameters $\{n, L\}$ and for a given operating point $\left\{V_{1}, V_{2}, P\right\}$.

\section{RESUlts}

The propsed modulation scheme is verified by means of a simulation employing GaN-Systems GS66516B HEMTs as semiconductor switches and an additional parasitic layout capacitance of $C_{\mathrm{par}}=100 \mathrm{pF}$. Evaluating (3) for a constant voltage of $V_{1}=400 \mathrm{~V}$ and an inductance value of $L=23 \mu \mathrm{H}$ 


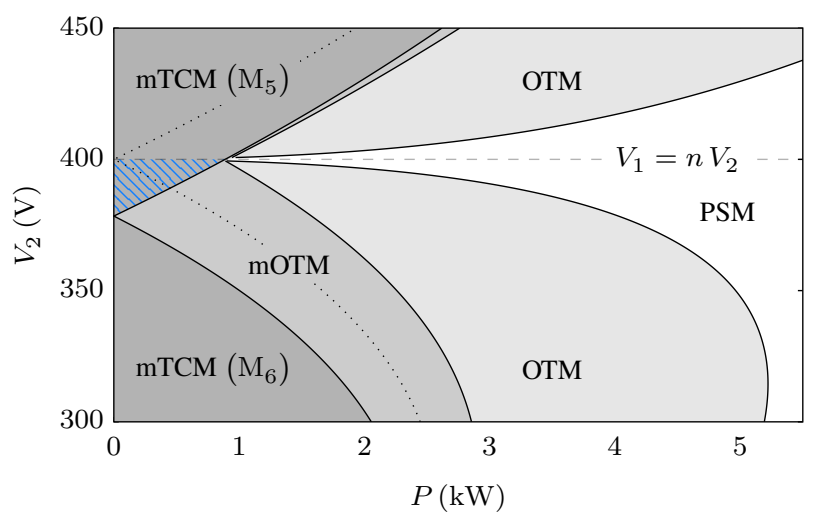

(a)

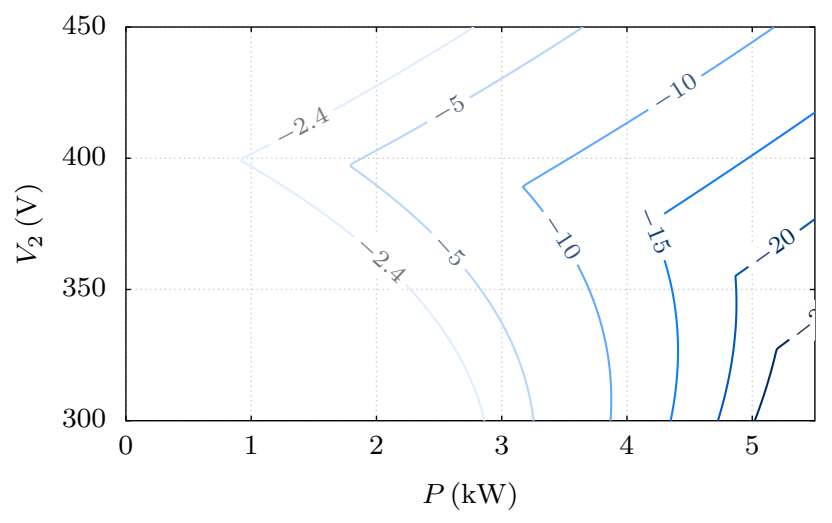

(b)

Fig. 5: (a) DAB operating modes employed for the proposed modulation scheme; contrary to standard TCM (dotted lines), $\mathrm{M}_{5}$ is also applied for $V_{1}>n V_{2}$. (b) switching currents obtained with the proposed modulation scheme which enable for full operating range primary-side ZVS.

points out, that a minimum current of $\left|I_{\mathrm{zvs}}\right|=2.4 \mathrm{~A}$ is required for a complete ZVS transition. The different modes generated by the proposed modulation scheme are presented in Fig. 5(a). As previously mentioned it becomes clear, that switching mode $\mathrm{M}_{6}$ is not only applied for operating points $V_{1} \geq n V_{2}$ for mTCM as it the case for standard TCM. Respective operating points are indicated by the shaded area in Fig. 5(a) and require the modified equations for the calculation of the optimal control variables.

Fig. 5(b) shows the current values for the primary-side switching instants obtained by the proposed modulation scheme. It becomes obvious that primary-side ZVS can be achieved throughout the entire operating range. The minimum current of $I_{\mathrm{zvs}}=-2.4 \mathrm{~A}$ is maintained for all operating points covered by the modulation schemes mTCM and mOTM, which proves the effectiveness of the proposed modulation strategy. For all OTM and PSM operating points this current at the respective switching instants is exceeded.

It should be mentioned that the trajectories of the modulation parameters become discontinuous at the border between mode $\mathrm{M}_{5}$ and mOTM for $V_{1}>n V_{2}$ (c.f. Fig. 5(a)). However, this exhibits only a minor drawback for the application at hand as a charger for high-voltage batteries of electric vehicles usually operates at medium to high power levels. If seamless transitions between the above mentioned switching modes are required and ZVS is not mandatory for low-power operating points, the requested switching currents in the respective regions of the operating range could be reduced e.g. according to

$$
I_{\mathrm{zvs}}(P)=I_{\mathrm{zvs}} \min \left(\frac{|P|}{1000}, 1\right) .
$$

\section{CONCLUSION}

In this paper, a procedure to analytically calculate the optimal modulation parameters for a dual active bridge converter has been presented, which inherently facilitates either primary or secondary-side ZVS in the full operating range. The modulation parameters are determined in such a way that the impressed current at the respective switching instants is high enough to guarantee an ideal ZVS transition. ZVS for the opposite bridge can still be achieved by adding an additional commutation inductor. Unlike existing approaches, analytical expressions have been derived which enable for a direct calculation of the respective modulation parameters without the need of preceding transformations applied to the converter model and/or additional changes in hardware.

\section{REFERENCES}

[1] R. M. Burkart and J. W. Kolar, "Comparative $\eta-\rho-\sigma$ Pareto Optimization of Si and SiC Multilevel Dual-Active-Bridge Topologies With Wide Input Voltage Range“, in IEEE Trans. on Power Electron., vol. 32, no. 7, pp. 5258-5270, July 2017.

[2] S. Inoue and H. Akagi, "Bidirectional DC-DC converter for an energy storage system with galvanic isolation“, IEEE Tran. Power Electron., vol. 22, no. 6, pp. 2299-2306, Nov. 2007

[3] M. Kasper, R. M. Burkart, G. Deboy and J. W. Kolar, "ZVS of Power MOSFETs Revisited“, IEEE Trans. Power Electron., vol. 31, no. 12, pp. 8063-8067, Dec. 2016.

[4] F. Krismer and J. W. Kolar, "Closed form solution for minimum conduction loss modulation of DAB converters", IEEE Trans. Power Electron., vol. 27, no. 1, pp. 174-188, Jan. 2012.

[5] M. Saegmueller, C. Hackl, R. Witzmann and R. Richter, "Analytic Solutions for Minimum Conduction Loss Modulation of Dual Active Bridge Converters Including Frequency Variation“, Proc. of 46th Annual Conference of the IEEE Industrial Electronics Society, pp. 4661-4666, Oct. 2020

[6] Z. Guo, "Modulation Scheme of Dual Active Bridge Converter for Seamless Transitions in Multiworking Modes Compromising ZVS and Conduction Loss“, IEEE Trans. Industrial Electron., vol. 67, no. 9, pp. 7399-7409, Sept. 2020.

[7] J. Everts, "Closed-Form Solution for Efficient ZVS Modulation of DAB Converters", in IEEE Trans. on Power Electron., vol. 32, no. 10, pp. 7561-7576, Oct. 2017. 